\title{
Laboreal
}

Volume 16 NN$^{\circ} 2$ | 2020

Programa de Pesquisa do Curso da Ação

\section{Impactos de la pneumónica en el mundo del trabajo : una visión desde los periódicos (1918-19)}

Impactos da pneumónica no mundo do trabalho : uma visão a partir de periódicos (1918-19)

Maria da Luz Sampaio

\section{OpenEdition}

\section{Journals}

Edición electrónica

URL: http://journals.openedition.org/laboreal/17426

DOI: $10.4000 /$ laboreal. 17426

ISSN: 1646-5237

Editor

Universidade do Porto

Referencia electrónica

Maria da Luz Sampaio, «Impactos de la pneumónica en el mundo del trabajo : una visión desde los periódicos (1918-19) », Laboreal [En línea], Volume 16 №2 | 2020, Publicado el 01 diciembre 2020, consultado el 13 diciembre 2020. URL : http://journals.openedition.org/laboreal/17426 ; DOI : https:// doi.org/10.4000/laboreal.17426

Este documento fue generado automáticamente el 13 diciembre 2020.

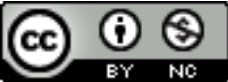

Laboreal está licenciado com uma Licença Creative Commons - Atribuição-NãoComercial 4.0 Internacional. 


\section{Impactos de la pneumónica en el mundo del trabajo : una visión desde los periódicos (1918-19)}

Impactos da pneumónica no mundo do trabalho : uma visão a partir de

periódicos (1918-19)

Maria da Luz Sampaio

\section{NOTA DEL EDITOR}

Traducción : Fernanda Romero [fernandaromero.trad@gmail.com]

\section{Prolegómenos a una breve incursión en la historia de la Pneumónica}

Entre 1918 y 1919, la humanidad fue víctima de una epidemia conocida internacionalmente como Spanish Influenza o Spanish Fluy, en Portugal, como Pneumónica (o Gripe Pneumónica). En mayo de 1918, la epidemia es declarada en España, se propaga en seguida por toda Europa y en pocas semanas se extiende a todos los continentes. En el mismo mes, la epidemia llegó a Portugal, afectando en primer lugar las regiones fronterizas, pero en pocos meses se extendió a los principales centros urbanos del país. A partir de entonces, fue posible identificar dos olas distintas de la gripe española : la primera, entre mayo y finales de julio, que se ha mantenido más o menos controlada, y la segunda ola, entre agosto y diciembre, que asumió efectos dramáticos y devastadores en una población poco informada y con escasa aplicación de las medidas de salud pública, haciendo inevitable una propagación que resultó implacable. 
2 A este panorama se aunaron los brotes de tifus, viruela y tuberculosis, que diezmaron los más débiles y desfavorecidos de la población. Para comprender lo que las poblaciones vivieron y que impacto tuvo la epidemia de Pneumónica o Influenza en el mundo del trabajo, necesitamos realizar una profunda incursión en los archivos, tanto de las empresas que existían en ese momento como de la prensa de esa época. Otra importante fuente para estos estudios sería el acceso a la documentación del Ministerio de Trabajo y de Previdencia Social. Esta investigación, que carece de un estudio y de un análisis de las fuentes exhaustivo y profundizado, presenta en este artículo algunos de sus resultados previos. Aunque sin la profundidad que desearíamos, registramos datos sobre los distintos sectores de actividad en la prensa durante el año de 1918, en particular en los periódicos O Comércio do Porto, A Capital y O Algarve (1918-19), disponibles en la Hemeroteca Digital, de entre una selección que se basó en la observación de las noticias producidas en tres lugares del país, en tres proyectos editoriales distintos. Buscamos encontrar en este estudio informaciones sobre la actuación de diversos sectores de actividad durante la incidencia de la Pneumónica y cuáles fueron los impactos en el mercado de trabajo y en la actividad de las empresas.

\section{Portugal en 1918 : el hambre, la guerra y los brotes epidémicos}

3 En 1918, Portugal era un país de carácter rural que se enfrentaba a una situación económica y financiera compleja - sufriendo el impacto del conflicto mundial - ya de sí suficientemente debilitada por una economía "dependiente del comercio exterior en materia de suministros de alimentos (cereales) y energía (carbón)" (Pires, 2018).

4 El armisticio, firmado el 11 de noviembre, y el fin de la guerra, en 1918, trajo los soldados de vuelta a casa, pero la gran mayoría regresó mutilada o sufriendo los efectos de los gases, un escenario al cual muchas mutualidades intentaron dar respuesta desarrollando campañas de apoyo. A la par de esta dura realidad, la guerra había traído también la falta de muchos productos extranjeros y, en Portugal, para hacer frente a la escasez de productos de primera necesidad, se crearon las Comisiones de subsistencias, controladas por el poder local, con la intención de asegurar el suministro de bienes alimenticios a través de estas.

5 La prensa de la época registraba con regularidad el papel de las Comisiones en la distribución de pan, azúcar y gasolina, entre otros productos, pero también las irregularidades cometidas por las panaderías y tiendas de ultramarinos que retenían a los productos para aumentar los precios (Pires, 2018). El hambre, la guerra y los brotes epidémicos serían, en este año de 1918, una trilogía fatal para los grupos más vulnerables de la población.

6 El periódico O Comércio do Porto, publicó, en septiembre de 1918, un artículo intitulado "A Influenza - Pneumónica", divulgando el documento que Ricardo Jorge había presentado al Consejo Superior de Hygiene y donde se relata la historia del surgimiento de la Influenza que afectó sobre todo a los cuarteles, principales focos de la epidemia, y de donde habrá salido para propagarse con rapidez, preferentemente a gente nueva (...) (O Comércio do Porto, 1918). Señala asimismo que "no se ofrece ninguna profilaxis efectiva y eficaz contra dicha epidemia que no sea la hygiene general y a asistencia a los afectados, preferiblemente en un Hospital de aislamiento" (O Comércio do Porto, 1918). 
7 Pese a los informes y advertencias de Ricardo Jorge, en la época Director General de la Salud, la realidad evidenciaba la falta de estructuras organizadas entre la población con el fin de promover la atención clínica y revertir la propagación del brote. La pandemia tenía, por lo tanto, muchos factores a su favor : en primer lugar, la guerra, después el hambre generalizado debido a la escasez de bienes alimenticios y de medicamentos $y$, para empeorar, la falta de condiciones de higiene y sanitarias de las casas y barrios donde vivía la población, sin agua corriente o saneamiento. En Oporto, la prensa registraba cómo la población de las ilhas (barrios obreros), en estos lugares húmedos e insalubres, era un foco de la enfermedad, que diezmaba fácilmente a familias enteras. A las corporaciones de bomberos les competía incinerar las pequeñas y rudimentarias casas, intentando así detener la propagación de la enfermedad. A esta gripe se sumó la "epidemia de tifus, que afectó particularmente a las zonas de Oporto y Espinho, diezmando a más de 2000 personas" (Sequeira, 2001, p. 50). El 15 de octubre, ante un pico del brote epidemiológico, el mismo periódico presenta el impacto de la pandemia en distintas zonas del norte del país, señalando el aumento de la tasa de mortalidad y, en las Notas por localidad, refiere que, "en Santo Thyrso se espera la reapertura de la fábrica de hilados", un sentimiento compartido por los más afectados, sobre todo los obreros fabriles y sus supervisores.

\section{Los impactos de la pandemia : entre la crisis y la voluntad de recuperación}

8 A lo largo de 1918, los periódicos analizados nos presentan la irregularidad del funcionamiento de las fábricas y de los servicios públicos, debido al fallecimiento de industriales y de los largos períodos de convalecencia de los empleados. En noviembre de 1918, el semanario O Algarve mencionaba que "la regularidad del telégrafo-postal, sobre todo de los servicios de correos, no ha podido ser restablecida porque la circulación ferroviaria sigue sin personal para desempeñar los distintos puestos de trabajo (...), una vez que la mortalidad por la pandemia y las largas convalecencias ocasionaron una gran escasez de empleados" (O Algarve, 17 noviembre 1918, p. 2).

9 La actividad industrial vive los impactos de la enfermedad, también por la falta de materias primas importadas, tales como carbón, harinas y algodón, entre otras, lo que ha afectado la producción y el suministro de productos de primera necesidad a las poblaciones. Las noticias y los artículos de opinión revelan que nos enfrentamos a una escasez prolongada, pero también a movimientos especulativos de comerciantes e industriales, que retenían los productos en los almacenes para así aumentar los precios. El 30 de enero de 1919, el periódico A Capital publica la noticia "La Refinaria Colonial, en Alcântara, tiene 200 toneladas de azúcar ya fabricado y 400 toneladas en la fábrica, careciendo ya de instalaciones para almacenar los sacos llenos" (A Capital, enero 1919, p.2). Otro lado de esta realidad nos releva que la vida en las empresas se estaba reorganizando frente a la pandemia, tal y como lo traduce el periódico O Algarve con la publicación de una protesta pública de la empresa Electro Moagem Lda., de Faro, contra las decisiones arbitrarias tomadas por el supervisor del granero municipal :

En esa época, todos nuestros empleados estaban enfermos, pero debido a la buena voluntad de nuestro gerente, la fábrica trabajó bajo su dirección y al final de 14 días laborales habíamos entregado al granero su respectiva harina. Fuimos 
personalmente pedirle a su Exa. más trigo, de aquel que había llegado a Faro (...). Nos dijo que la "fábrica grande" empezaría a trabajar y que se había comprometido a entregar a esa fábrica todo el trigo que llegase (...). Le hicimos ver a su Exa. que no deseábamos que nos entregara todo el trigo, sino este prorrateado en la proporción de la producción de cada fábrica, en armonía con el decreto 4.638 del 13 de junio, pero su Exa. no se movió y el hecho es que la dicha fábrica grande (...) hace casi ocho días que tiene en sus almacenes 4 a 5 vagones de trigo para moler (...), sin que se sepa exactamente cuándo empezará a trabajar" (O Algarve, 17 de noviembre de 1918, n 556, p. 2).

Las situaciones de crisis y enfermedad ponían en evidencia la arbitrariedad de los responsables por la gestión de los bienes esenciales y la permisividad del poder local de cara a los movimientos especulativos. Por otro lado, también surgieron otras oportunidades de negocio, como fue el caso de la industria conservera y de las compañías de seguros (O Algarve, 16 de junio de 1918).

11 A la par de dichas oportunidades de negocio en ciertos sectores, la pandemia vino a imponer restricciones de movimientos, con la finalidad de prohibir eventos que favoreciesen la concentración de personas y animales. Un ejemplo de esta situación ha sido la suspensión, o incluso la prohibición, de las ferias de otoño. El periódico O Algarve publicó, en octubre de 1918, que la Feria de Faro no se realizaría como consecuencia de la propagación de la epidemia, pero si "las Ferias no fueron consentidas, (...) las gentes, sin embargo, que concurrieron, hicieron la feria de ganado en un campo fuera de la cuidad" (O Algarve, 18 de octubre, p. 2).

12 En lo que respecta al comercio, a lo largo de la epidemia, las tiendas se mantuvieron abiertas, renovaron sus escaparates con la llegada de los productos y de las nuevas colecciones, dando signos de continuidad de la actividad, a pesar de la lista de fallecimientos de comerciantes y de empresarios de la Plaza. El periódico A Capital publica regularmente noticias sobre los productos de Armazéns do Chiado, refiriendo la variedad de artículos que allí se pueden encontrar.

13 La vida artística también da muestras de vitalidad, todos los periódicos anuncian conciertos, recitales y espectáculos de teatro. Es más, este parece ser un sector que fomenta las salas llenas de espectadores, un local donde la pandemia (aparentemente) parecía haber sido olvidada.

14 Los grandes centros urbanos se vieron muy afectados por los brotes pandémicos, pero también lo fueron algunas regiones donde había una mayor concentración de trabajadores. La prensa informó también que en Felgueiras había muchas familias afectadas por la gripe ; en Matosinhos, el número de casos aumentaba diariamente, por lo que se rezaba al Senhor de Matosinhos para poner fin a la pandemia; en Braga, la epidemia en el mes de octubre registraba diariamente 14 a 15 fallecimientos y en el Hospital de S. Marcos, el personal de enfermería cayó enfermo, por lo que solicitaron personal militar para su sustitución; en Santa Cruz do Douro, el gran número de trabajadores que se desplazaron a las vendimias regresaron atacados por la enfermedad... "Y ya se registraron bastantes fallecimientos, pero solo que aquellos que no han recibido atención médica" (o Commércio do Porto, 1918, p. 1).

El contagio de la gripe afectaba a los trabajadores, en todas las actividades, desde los trabajadores agrícolas e industriales hasta el personal clínico de los hospitales, diezmando familias y fragilizando aún más los trabajadores que estaban imposibilitados 
de obtener sus ingresos. En la prensa, las listas de obituarios y el nombramiento del fallecimiento de bienhechores eran constantes, bien como de prominentes industriales y empresarios o de miembros de sus familias.

El número de muertes causadas por la enfermedad sigue todavía por determinar con rigor, pero las últimas investigaciones de Rebelo-de-Andrade y Felismino (2018), apuntan a 117764 víctimas mortales en Portugal, considerando todas las infecciones respiratorias agravadas (gripe, tuberculosis pulmonar, bronquitis aguda y crónica, neumonía). Según los estudios de Abreu y Serrão (2018), la gripe, en Portugal, "habrá infectado a entre una quinta y una tercera parte de los cerca de seis millones que componían entonces la población residente (...). Afectó particularmente a la población en edad laboral, de entre 20 y 40 años, reforzando así su impacto en la economía, en el mercado laboral, en la fertilidad, en la vida familiar y en la organización social en general" (p.9). Estas cifras demuestran la vulnerabilidad de la población frente a la epidemia, la falta de medidas de protección y de servicios de salud que actuasen para frenar la proliferación de los brotes y que hubiesen, en esa época, permitido bajar las tasas de letalidad de las poblaciones.

\section{BIBLIOGRAFÍA}

Arquivo Municipal de Gaia/Gisa - Jornal o Commércio do Porto de 1918 e 1919.

Abreu, L., \& Serrão, J. V. (2018). Revisitar a pneumónica de 1918-1919 : introdução Dossier : Revisitar a Pneumónica de 1918-1919. Ler História, 73, 2018, 9-19.

Hemeroteca Digital - A Capital , Diário Republicano da Noite, 1918, 19. Disponível em http:// hemerotecadigital.cm-lisboa.pt/Periodicos/ACapital/ACapital.HTM. Consultado em 02- 08 de novembro de 2020 .

Hemeroteca digital - O Algarve, Semanário independe 1918-19. Disponível em http:// hemerotecadigital.cm-lisboa.pt/Periodicos/ACapital/ACapital.HTM. Consultado em 02- 08 de novembro de 2020 .

Pires, A. P. (2018). Lisboa e a grande guerra : subsistências e poder municipal, 1916-1918. In Ler História, 73, 2018. http://journals.openedition.org/lerhistoria/4267, consultado no dia 22 setembro 2020.

Rebelo-de-Andrade, H., \& Felismino, D. (2018). A pandemia de gripe de 1918-1919 : um desafio à ciência médica no princípio do século XX. In Ler História , 73, http://journals.openedition.org/ lerhistoria/4070, consultado no dia 22 setembro 2020.

Sequeira, Á. (2008). A Pneumónica, Spanish Influenza. História da Medicina, 8, 1, 40-55. 


\section{AUTOR}

\section{MARIA DA LUZ SAMPAIO}

HTC- FCSH Universidade Nova de Lisboa, Faculdade de Ciências Sociais e Humanas, Universidade Nova de Lisboa, Avenida de Berna 26 C, 1069-161 Lisboa

mluzsampaio@gmail.com ; mluzsampaio@fsch.unl.pt 